\title{
P I I- 19. Altered mucosal distribution of SIV-specific T cells in Rhesus macaques infected with the live-attenuated Rev-independent SIV
}

\author{
A Valentin ${ }^{* 1}$, A von Gegerfelt ${ }^{1}$, C Alicea ${ }^{2}$, V Patel ${ }^{1}$, ML Marthas ${ }^{3}$, K Van \\ Rompay $^{3}$, GN Pavlakis ${ }^{1}$ and BK Felber ${ }^{2}$
}

\author{
Address: ${ }^{1}$ Center for Cancer Research, HRS, VB, NCI-Frederick, Frederick, USA, ${ }^{2}$ HRPS, VB, NCI-Frederick, Frederick, USA and ${ }^{3}$ California National \\ Regional Primate Research Center, Davis, CA, USA \\ * Corresponding author
}

from AIDS Vaccine 2009

Paris, France. 19-22 October 2009

Published: 22 October 2009

Retrovirology 2009, 6(Suppl 3):PI64 doi:10.II86/I742-4690-6-S3-PI64

This abstract is available from: http://www.retrovirology.com/content/6/S3/PI64

(c) 2009 Valentin et al; licensee BioMed Central Ltd.

\section{Background}

Infection of rhesus macaques with live-attenuated SIV strains provides the best known protection against challenge with pathogenic SIV. A cohort of 10 Indian rhesus macaques infected by Rev-independent live-attenuated SIV was monitored for up to 10 years. After the initial acute phase, virus replication was controlled and plasma viral loads were persistently below the threshold of the assay. None of the animals showed any signs of immune dysfunction.

\section{Methods}

SIV-specific T cells were monitored in peripheral blood, bronchoalveolar lavage (BAL) and rectal biopsies by multiparametric flow cytometry after stimulation with gag or env peptide pools. The results were compared with those obtained from similar samples taken from macaques infected with the pathogenic SIVmac251.

\section{Results}

Macaques infected with SIVmac251 had significantly higher SIV-specific T cells in BAL than blood ( 5 to 10 -fold higher). No responses were detected in gastrointestinal samples. In contrast, animals infected with the Rev-independent SIV had similar or lower T cell responses in BAL compared to blood. In addition, the presence of SIV-specific T cells after peptide stimulation was detected in rectal samples from $60 \%$ of the animals infected with the atten- uated SIV, and these responses were consistently higher than those present in BAL. Responses against env were mediated preferentially by CD8+ T cells with effector phenotype (CD28-CD95+CCR7-) whereas gag responses were mediated mainly by CD4+ T cells. Antigen-specific T cells in both mucosal compartments were characterized by the lack of CCR7 and CD45RA.

\section{Conclusion}

The distribution of SIV-specific T cells in mucosal sites is different in macaques infected with WT or with the liveattenuated Rev-independent SIV. Preservation of cellular immunity in the GALT could be related to the efficient control of viral replication associated with live-attenuated SIV infection. 